\title{
Analysis of contact between barrel and projectile of a rifle based on finite element method
}

\author{
Benjun Zhang ${ }^{\mathrm{a}}$, Guangsheng Liu, Youmin Wei and Yue Li \\ Shijiazhuang Mechanical Engineering College, Shijiazhuang, Hebei, China
}

\begin{abstract}
To analysis of contact interaction between barrel and projectile, its finite element calculation formulas were deduced on the base of contact theory. By using the new method and finite element model, the contact interaction between barrel and projectile of a rifle was calculated. The calculation results show that the barrel and projectile were plastic deformation, especially the equivalent stress of projectile has been in strength limit region, and this is the main reason for embed copper during firing. So, the structure of barrel rifling and projectile should be optimized. The optimization process can be guided by the new method, and a computing platform for the process was provided by the established model.
\end{abstract}

Keywords: finite element, contact theory, equivalent stress, barrel rifling, projectile.

\section{Introduction}

Rifle is used to launch projectile and hit target accurately, the projectile is driven in the barrel by the high temperature and high pressure propellant gas. During the movement process, and the barrel rifling influence each other. Recoil is not in position is occurred by using a general bullet, and the main reason is that the gas port is jammed by embed copper. Embed copper can be appear by match between barrel and projectile is not reasonable will, and to optimize the matching needs to analysis of contact interaction between barrel and projectile.

The most widely used of relationship between gun and projectile is taking the action of projectile as an equivalent force, and then research on firearms dynamics characteristics, human-gun interactions and structure optimization [1-3]. There are also some scholars take the action of projectile as an equivalent force to research strength of the barrel force by considering the thermal [4-5]. The two kinds of situations are not considered the projectile body during modeling and calculating. The forcing cone model by considering projectile body was built by Lu Ye by using LS-DYNA, which is used to optimize the angle of the forcing cone in order to improve the firing accuracy, but the contact interaction between barrel and projectile was not researched systematically[6]. In fact, the contact between barrel and projectile is a finite element problem which involves the contact nonlinearity. For the contact nonlinear problem, many people have been applied in different fields [7-8].

To research contact interaction between barrel and projectile, its finite element calculation formulas were deduced on the base of contact theory, and the stress state of barrel and projectile was calculated, which can provide guidance for the improvement of the rifling and projectile. 


\section{Basic assumption}

During the firing process, the interaction between rifles and projectile is quite complex, there are a lot of force action such as bore force, force of interference fits and thermal stress, also may be a chemical change takes place. So considering all the circumstances during analysis contact interaction between barrel and projectile is not realistic, and then the following assumptions were put forward.

(1) Only considering the bore force and force of interference fits, without considering the thermal stress and chemical reaction.

(2) Considering the plastic deformation of the barrel and projectile, without considering problem of failure.

(3) The calculation process is equivalent to quasi-static process.

\section{Contact interaction calculation}

The projectile and barrel rifling squeeze each other under the action of bore force during firing, such as figure 1 shown. The contact parts of barrel rifling and projectile was bear under the action squeeze pressure, and the parts behind contact parts was bear bore force, which is shown in figure 2.

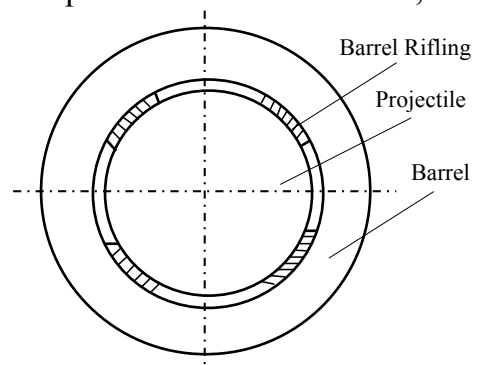

Figure 1. Schematic of barrel and projectile contact.

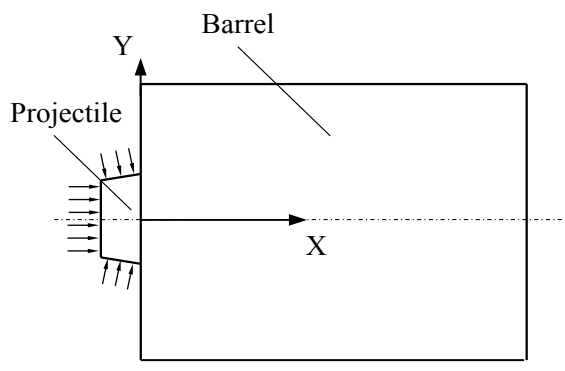

Figure 2. Schematic of force of projectile.

The global coordinate system was set up which was shown in Figure 2, the forward of $Z$ axis was determined by the right rule. Take $S^{C}$ as the contact interface between barrel rifling and projectile, $S^{A}$ as the contact interface on barrel rifleand $S^{B}$ as the contact interface on projectileMutually perpendicular unit vectors $\boldsymbol{e}_{1}$ and $\boldsymbol{e}_{2}$ is taken out in a tangent plane of point $\mathrm{P}$ which is any point in $S^{B}$, and normal unit vector $\boldsymbol{e}_{3}$ is also taken out, then local coordinate system is established. Point $\mathrm{Q}$ is the contact point with $\mathrm{P}$ in the $S^{A}$.

There should be non-penetration in contact problem, and the contact force should be pressure. According the requirements, normal condition formulas was obtained.

$$
g_{3}=\left(\boldsymbol{u}^{A}-\boldsymbol{u}^{B}\right) \boldsymbol{e}_{3}-\left(\boldsymbol{x}^{A}-\boldsymbol{x}^{B}\right) \boldsymbol{e}_{3}=u_{3}^{A}-u_{3}^{B}+\bar{g}_{3} \geq 0
$$




$$
F_{3}^{A}=-F_{3}^{B} \geq 0
$$

$\boldsymbol{u}^{A}$ and $\boldsymbol{u}^{B}$ were the displacement vectors in the local coordinate system of $\mathrm{P}$ and Q. $\boldsymbol{x}^{A}$ and $\boldsymbol{x}^{B}$ were the initial coordinate vectors in the local coordinate system of $\mathrm{P}$ and $\mathrm{Q} . u_{r}^{i}$ was the component displacement of $\boldsymbol{u}^{i}$ in $\boldsymbol{e}_{r}$ direction. $\bar{g}_{3}$ was the initial penetration in local coordinate system. $F_{r}^{i}$ was the component force of $\boldsymbol{F}^{i}$ in $\boldsymbol{e}_{r}$ direction. $i=A, B, r=1,2,3$.

Because there is no sliding in contact, tangential contact formula and judgment state formula were obtained by using Coulomb friction model.

$$
\begin{aligned}
& u_{1}^{A}-u_{1}^{B}=u_{2}^{A}-u_{2}^{B}=0 \\
& \sqrt{\left(F_{1}^{i}\right)^{2}+\left(F_{2}^{i}\right)^{2}} \leq \mu F_{3}^{i}
\end{aligned}
$$

$\mu$ was friction coefficient. Taken contact force as a surface force boundary conditions in barrel and projectile, and formulas (5) was obtained by using principle of virtual displacement. Formulas (6) was expression of $W_{C}$ which is the virtual work done by contact force.

$$
\begin{gathered}
\int_{V} \sigma^{T} \delta \varepsilon d V-W_{C}=0 \\
W_{C}=\int_{S_{C}} \boldsymbol{F}^{A} \delta \boldsymbol{u}^{A} d s+\int_{S_{C}} \boldsymbol{F}^{B} \delta \boldsymbol{u}^{B} d s=\int_{S_{C}} \boldsymbol{F}^{A}\left(\delta \boldsymbol{u}^{A}-\delta \boldsymbol{u}^{\boldsymbol{B}}\right) d s
\end{gathered}
$$

Formula (7) shows functional which contains contact interface, and which was obtained by using lagrangian multiplier method. Formula (8) shows $\Pi_{C P}$ which is additional functional for the introduction of contact conditions.

$$
\begin{gathered}
\Pi=\Pi_{u}+\Pi_{C P} \\
\Pi_{C P}=\int_{S_{C}}\left[\lambda_{3}\left(u_{3}^{A}-u_{3}^{B}+\bar{g}_{3}\right)^{2}+\lambda_{1}\left(u_{1}^{A}-u_{1}^{B}\right)^{2}+\lambda_{2}\left(u_{2}^{A}-u_{2}^{B}\right)^{2}\right] d
\end{gathered}
$$

$\lambda_{1} \lambda_{2}$ and $\lambda_{3}$ are lagrangian multipliersTaken $\boldsymbol{u}$ variational in formula (8) and then:

$$
\left(\delta \Pi_{C P}\right)_{u}=\int_{S_{C}}\left[\lambda\left(\delta \boldsymbol{u}^{A}-\delta \boldsymbol{u}^{B}\right) d s\right.
$$

Taken formula (8) into formula (7), formula (10) was obtained for $\delta \Pi=0$ by comparing with formula (5).

$$
W_{C}=-\left(\delta \Pi_{C P}\right)_{u}=\int_{S_{C}}-\left[\lambda\left(\delta \mathbf{u}^{A}-\delta \mathbf{u}^{B}\right) d s\right.
$$

At the same time, $\left(\delta \prod_{C P}\right)_{\lambda}=0$ can be obtained by randomness of $\delta \lambda$, and then formula (11) was obtained, and then formula (11) was also obtained.

$$
u_{3}^{A}-u_{3}^{B}+\bar{g}_{3}=0, \quad u_{1}^{A}-u_{1}^{B}=0, \quad u_{2}^{A}-u_{2}^{B}=0
$$




$$
F_{r}^{A}=-\lambda_{r}, \quad F_{r}^{B}=\lambda_{r}, \quad r=1,2,3
$$

Barrel and projectile were discretized, and taken $\boldsymbol{u}_{P}$ as displacement of node $\mathrm{P}, \boldsymbol{u}_{Q}$ as $\mathrm{Q}$ which contact with $\mathrm{P} . \boldsymbol{u}_{Q}$ can be obtained by the nodes displacement of element.

$$
\boldsymbol{u}_{Q}=\sum_{i=1}^{m} \boldsymbol{N}_{i}\left(\xi_{Q}, \eta_{Q}\right) \boldsymbol{u}_{i}^{Q}
$$

$\boldsymbol{N}_{i}$ was the shape function of element. $\left(\xi_{Q}, \eta_{Q}\right)$ was natural coordinates of node $\mathrm{Q}$ in the element. $\boldsymbol{u}_{i}^{Q}$ was nodes displacement of element. $m$ was number of nodes of element. Relative displacement of P and Q can be obtained by using formula (13).

$$
\boldsymbol{u}_{p}-\boldsymbol{u}_{Q}=N_{c} \boldsymbol{u}_{c}, \quad N_{c}=\left[\begin{array}{lllll}
\boldsymbol{I}_{3 \times 3} & -N_{1} & -N_{2} & \ldots & -N_{m}
\end{array}\right], \quad \boldsymbol{u}_{c}=\left[\begin{array}{lllll}
\boldsymbol{u}_{P}^{T} & \boldsymbol{u}_{1}^{T} & \boldsymbol{u}_{2}^{T} & \ldots & \boldsymbol{u}_{m}^{T}
\end{array}\right]^{T}
$$

$\boldsymbol{\theta}$ is the transfer matrix of Global coordinate system and local coordinate system, and then:

$$
\boldsymbol{u}_{p}-\boldsymbol{u}_{Q}=\boldsymbol{N}_{c} \boldsymbol{u}_{c}, \boldsymbol{u}^{A}-\boldsymbol{u}^{B}=\boldsymbol{\theta}^{T}\left(\boldsymbol{u}_{p}-\boldsymbol{u}_{Q}\right)=\boldsymbol{\theta}^{T} \boldsymbol{N}_{c} \boldsymbol{u}_{c}
$$

Discrete form of virtual work about contact force can be obtained after discretized barrel and projectile.

$$
W_{C}=\sum_{k=1}^{n_{C}}\left(W_{C}\right)_{k}=\sum_{k=1}^{n_{C}}\left(\delta \boldsymbol{u}_{c}^{T} \boldsymbol{N}_{c}^{T} \boldsymbol{\theta} \boldsymbol{F}^{A}\right)_{k}
$$

$n_{C}$ was contact pair number. Based on formula (16), $\left(\boldsymbol{Q}_{c}\right)_{k}$ which is node equivalent contact force vector of the first $\mathrm{k}$ contact point was obtained.

$$
\left(Q_{c}\right)_{k}=\left(N_{c}^{T} \theta F^{A}\right)_{k}
$$

Formula (18) was obtained by taking formula (15) into formula (13).

$$
\left(\boldsymbol{\theta}^{T} \boldsymbol{N}_{c} \boldsymbol{u}_{c}\right)_{k}=-\left[\begin{array}{lll}
0 & 0 & \bar{g}_{3}
\end{array}\right]^{T}=(-\overline{\boldsymbol{g}})_{\mathrm{k}}
$$

Formula (19) was obtained by integrating all contact points, and the parameter values of the formula were shown in formula (20). Then the finite element calculation formulas were obtained.

$$
\begin{aligned}
& \boldsymbol{Q}_{c}=-\boldsymbol{K}_{c \lambda} \lambda, \quad-\boldsymbol{K}_{c \lambda} \boldsymbol{u}_{c}=-\overline{\boldsymbol{g}}
\end{aligned}
$$

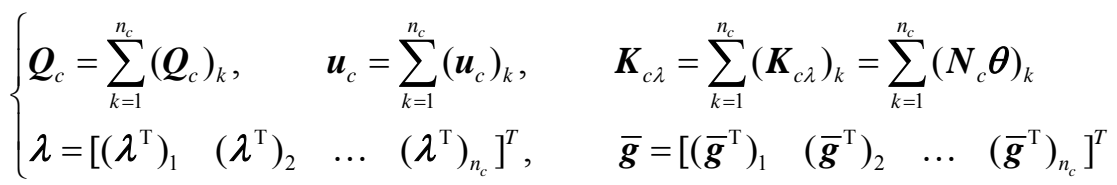

$$
\begin{aligned}
& {\left[\begin{array}{cc}
\boldsymbol{K} & \boldsymbol{K}_{c \lambda} \\
\boldsymbol{K}_{c \lambda}{ }^{T} & \mathbf{0}
\end{array}\right]\left(\begin{array}{l}
\boldsymbol{u} \\
\lambda
\end{array}\right)=\left(\begin{array}{c}
\boldsymbol{Q}_{L} \\
-\overline{\boldsymbol{g}}
\end{array}\right)}
\end{aligned}
$$


$\boldsymbol{K}$ is the stiffness matrix which contains the material nonlinearity. It can be obtained by using the construction method of the elastic plastic stiffness matrix according to the literature [9]. $\boldsymbol{Q}_{L}$ was equivalent external force vector. In the above formula, due to the nonlinearity of the material, formula (21) contains nonlinear term. Linearization needs to be carried out during calculating, and the formulas can be solved by using the numerical method, then equivalent nodal force was obtained.

\subsection{Calculation example}

Poor accuracy and embed copper were happened during firing a general bullet of a rifle, so optimizing computation of barrel rifling and projectile should be carried out. According to the above theory, combined with ANSYS software, contact interaction of barrel rifling and projectile of the rifle was calculated, which provides theoretical basis and platform for optimization calculation.

\subsection{Building finite element}

Entity model of barrel and projectile was established by using ANSYSY based on production drawing. Bilinear elastic-plastic material model and 8-nodes solid element were selected to mesh the model. Figure 3 shows the result. Face to face contact element which shows in figure 4 was selected to deal with contact element, and the boundary condition of the model is fixed outer surface of barrel.

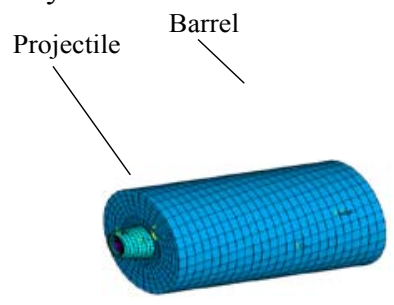

Figure 3. Finite element model of barrel and projectile.

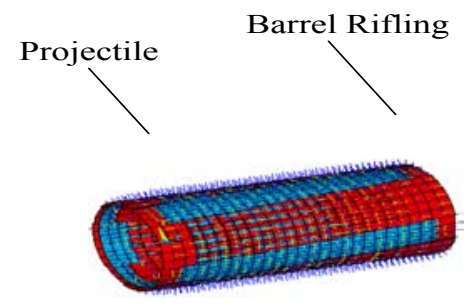

Figure 4. Model of contact element.

\subsection{Calculation result}

Contact interaction between barrel and can be calculated by given equivalent external force to the finite element model. Equivalent stress of barrel and projectile were obtained by using post processing of ANSYS, where are show in figure 5 and figure 6. Quarter of barrel was selected to analysis equivalent stress expediently in figure 5 .

The follow conclusions can be obtained by figure 5 and figure 6 .

(1) Maximum equivalent stress of projectile has been in strength limit region of the material, so partial material of projectile shell will remain in the bore, and this is the main reason for embed copper during firing.

(2) The maximum equivalent stress of barrel is beyond the yield limit, but less than the ultimate strength, so it can be determined that plastic deformation was occurred. The equivalent stress of 
Segmentation surface of barrel rifling was significantly greater than that in other parts, which may be caused by stress concentration.

(3) Compared with elastic deformation, the plastic deformation of projectile is obviously larger than that of elastic deformation, which shows that plastic deformation is the main deformation state during firing. So, the traditional bullet strength checking theory is limitation.

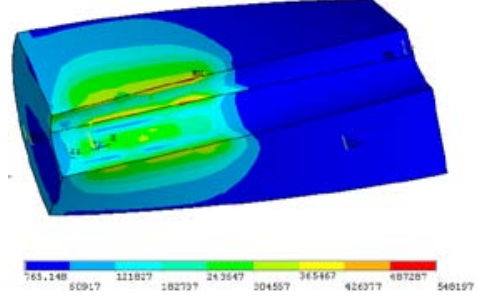

Figure 5. Equivalent stress of a quarter barrel.

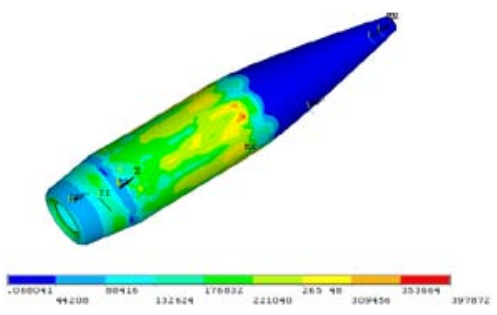

Figure 6. Equivalent stress of projectile.

\section{Conclusions}

To analysis of contact interaction between barrel and projectile, its finite element calculation formulas were deduced on the base of contact theory. By using the new method and finite element model, the contact interaction between barrel and projectile of a rifle was calculated. The calculation results show that the barrel and projectile were plastic deformation, especially the equivalent stress of projectile has been in strength limit region, and this is the main reason for embed copper during firing. So, the structure of barrel rifling and projectile should be optimized. The optimization process can be guided by the new method, and a computing platform for the process was provided by the established model.

\section{References}

1. LI Tao, WANG Ruilin, ZHANG Junnuo, et al. Simulation of Coupled Rigid and Flexible Multi-body Dynamics on Gatling Gun [J]. Journal of system Simulation, 2013, 25(6): 1382-1387.

2. Choi Young-Jin, Lee Young-Shin, Lee Se-Hoon, et al. Impact path analysis of human body with three typical shooting postures [J]. Key Engineering Materials, 2006, 326-328(1):899-902.

3. ZHANG Benjun, WANG Ruilin, LI Yongjian, et al.. Structural optimization for a machine-gun mount based on BP neural network and genetic algorithm [J]. Journal of Vibration and Shock, 2011, 30(1):142-144.

4. Wu Yong-hai, Xu Cheng, Zhang Hai-bing. Dynamic Response Analysis of Transient Thermo elasticity Couple of a Large-caliber Machine Gun Barrel [J]. Journal of Ballistics, 2006, 18(4): 16-20.

5. Yao Jian-jun, Xu Cheng, Guo kai. The Analysis to Thermal-stress Coupled Field in the Barrel of Rapid Fire Weapon [J]. Gun Launch \& Control Journal, 2001, (7):36-39.

6. Lu Ye, Zhou Ke-dong, He Lei, et al. Influence of Structure Parameters of Forcing Cone on Small Arms Interior Ballistics During Engraving [J]. ACTA ARMAMENTRAII, 2015, 36(7):1363-1369.

7. Shi Jin-yu. Mechanical Characteristics and Energy Analysis of a Pre-stressed Hydraulic Press 
Frame By Contact Finite Element Method [J]. Journal of Mechanical \& Electrical Engineering, 2014, 31(3): 354-357.

8. Zhang Ben-jun, Wang Rui-lin, Li Yong-jian, et al. Modeling and Analysis of Bullet Discarding Process of Open Link Chain [J]. ACTA ARMAMENTRAII, 2011, 32(12):1531-1534.

9. Wang Xu-cheng. Finite element method [M]. Beijing: Tsinghua University Press, 2003: 666-700.

10. Zhang Lei. Design and Research of Barrel Rifling Parameter Based on the Finite Element Theory [D]. Shijiazhuang: Ordnance Engineering College, 2013. 